\title{
Determinación Experimental y Contraste Numérico de la Resistencia Térmica de un Muro de Bloques de Concreto Hueco
}

Ana C. Borbón, Rafael E. Cabanillas y Jesús B. Pérez

División de Ingeniería, Universidad de Sonora, Blvd. Luis Encinas y Rosales, CP 83000, Hermosillo, Sonora-México (e-mail: acborbon@dicym.uson.mx; rcabani@iq.uson.mx; benito@dicym.uson.mx)

Recibido Ene. 07, 2010; Aceptado Feb. 22, 2010; Versión Final recibida Abr. 09, 2010

\begin{abstract}
Resumen
Se presenta el diseño y construcción de un dispositivo de pruebas para obtener la resistencia térmica de un muro de bloques de concreto hueco, cuyo funcionamiento se basa en el principio de la norma internacional denominada placa caliente protegida (guarded hot plate). Se describe el procedimiento constructivo y la puesta en marcha del dispositivo, reproduciendo las condiciones de operación para edificaciones ubicadas en clima cálido seco y se determina la resistencia térmica en forma experimental y numérica. Los resultados se obtienen aplicando diferencias de temperatura controlada en ambos lados de la pared, generando una zona de enfriamiento y otra de calentamiento, hasta alcanzar el estado estable. Tanto en los resultados experimentales como en los numéricos se observa, variaciones en el valor de la resistencia térmica que va desde 0.15 hasta 0.19 ${ }^{\circ} \mathrm{Cm} 2 / \mathrm{W}$. Los valores de la resistencia térmica fueron ajustados a una expresión empírica en función de la diferencia de temperaturas entre las paredes y de la temperatura promedio del muro.
\end{abstract}

Palabras clave: resistencia térmica, muro de bloque, estado estable, conjunto experimental.

\section{Experimental Determination and Numerical Contrast of Thermal Resistance in a Hollow Concrete Block Wall}

\begin{abstract}
This paper presents design and construction of an experimental test to determine the thermal resistance of a hollow concrete blocks wall, according to the international standard known as guarded hot plate. The construction procedure and implementation of the device are described, reproducing operations conditions for buildings located in warm dry climate, and the thermal resistance was experimentally and numerically determined. The results were found by applying controlled temperature differences on both sides of the wall between heating and cooling temperature zones, in steady state conditions. Experimental and numerical results show variations in the value of thermal resistance, ranging from 0.15 to $0.19{ }^{\circ} \mathrm{C} \mathrm{m} 2 / \mathrm{W}$. Values of the thermal resistance were fitted to an empirical expression as function of the temperature difference between the walls and the average temperature of the wall.
\end{abstract}

Keywords: thermal resistance, block wall, stable state, experimental test 


\section{INTRODUCCIÓN}

El estudio de propiedades térmicas en componentes constructivos en México es escaso, sobre todo si se trata de estudios experimentales. No existe una normatividad aplicada a reglamentos que exija a los constructores locales, respetar un mínimo de valores de resistencia térmica en los materiales de construcción. Se requiere conocer las propiedades termofísicas de componentes que se utilizan en la edificación, tales como bloques, tabiques y ladrillos; de configuración huecos y macizos, así como de diferentes geometrías y dimensiones, los cuales se utilizan para la elaboración de muros, en diferentes condiciones de operación, dependiendo del lugar y la zona climática donde se emplazan. Para el caso de climas extremos, algunos materiales experimentan comportamientos poco favorables si se trata de mantener, zonas dentro del confort térmico humano en las edificaciones. De los distintos materiales que se utilizan para la elaboración de muros, existen diferencias en cuanto a sus propiedades térmicas. El bloque de concreto hueco, es ampliamente utilizado en la construcción de muros de vivienda en serie en México; diversos estudios han mostrado, que tiene una resistencia térmica baja en relación con otros materiales y requiere climatización artificial con altos consumos de energía eléctrica. A pesar de esta limitante, el bloque de concreto es utilizado ampliamente dado que representa una forma económica de construir.

De acuerdo a la demanda de este material y el poco conocimiento que se tiene de sus propiedades térmicas, al ser integrado como componente de sistemas de muros en vivienda, se plantea hacer estudios experimentales, y evaluar el desempeño real. Para esto se ha diseñado y construido un muro de pruebas elaborado con bloques de concreto hueco, para la medición de variables termofísicas, para calcular su Resistencia Térmica.

El objetivo de este trabajo es el diseño, construcción y puesta en marcha de un dispositivo experimental que permita medir las variables y calcular la resistencia térmica de manera experimental, considerando las variaciones que esta tiene para diferentes $\Delta T$ y validar el modelo numérico de transferencia de calor, que simula el comportamiento de la transferencia de calor en el muro, y así proponer una expresión empírica para el cálculo de la resistencia térmica en muros de bloque hueco de 12x 20 × $40 \mathrm{~cm}$, que se fabrica en la región Noroeste de México, en función de los diferenciales de temperatura entre el lado interior y exterior del muro y de la temperatura promedio entre estas, bajo condiciones de operación de clima cálido extremoso.

En la bibliografía se encuentran trabajos reportados como el de Ossama y Murali (1994), donde se hace un estudio experimental en muros de bloque de concreto hueco, obteniendo valores de resistencia térmica de $0.36{ }^{\circ} \mathrm{Cm}^{2} / \mathrm{W}$, utilizando la técnica de placa caliente en bloques con cavidades; Aviram et al. (2001), muestra un estudio experimental de un muro de $1.2 \times 1.2 \mathrm{~m}$, utilizando la técnica de caja guardada, tomando en cuenta el efecto convectivo de las cavidades. Anderson (1981) realizó un estudio teórico, utilizando programación con elemento finito en dos dimensiones, en muros elaborados con bloques huecos de concreto, donde encontró valores de la resistencia alrededor de $0.25^{\circ} \mathrm{Cm}^{2} / \mathrm{W}$. En un estudio teórico para ladrillos de terracota huecos se muestra que la resistencia térmica disminuye con respecto al aumento de los diferenciales de temperatura (Vasile et al., 1998). Otros estudios teóricos como el de Chena y Wilson (2007) que presentan un análisis de la transferencia de calor con elemento finito en estado transciente. J. del Coz et al. (2006) presentan un estudio con elemento finito para encontrar las ecuaciones de transferencia de calor de bloques huecos, y el de Kossecka y Kosny (2004) donde utilizan códigos computacionales para diferencias finitas. En estudios experimentales se ha podido mostrar que los flujos de calor disminuyen al aumentar la razón de aspecto de la cavidad hueca, esto debido a que se frena la convección lo que tiene como consecuencia que se incremente la resistencia térmica (Aviram et al., 2001;Lacarriere et al., 2006). Entre los esfuerzos por reducir la transferencia de calor en muros de bloques se han probado mezclas con cemento aligerado aumentando la resistencia térmica de 0.1 hasta 0.24 ${ }^{\circ} \mathrm{Cm}^{2} / \mathrm{W}$ (Al-Jabri et al., 2004). No se encontraron estudios que reporten casos de grandes diferenciales de temperatura en resultados horarios y sus variaciones. Los reportes de los valores de $R$ varían de acuerdo a las características de los materiales y a las condiciones de operación dependiendo del medio donde se emplazan. 


\section{MODELO TEÓRICO}

Como antecedente a este trabajo experimental se retoman los resultados de un análisis teóriconumérico de transferencia de calor, unidireccional, en estado estable, donde se evaluó la resistencia térmica $(R)$ y el flujo de calor $(q)$ de un muro de bloques por $\mathrm{m}^{2}$ de superficie. El citado estudio está referenciado a un clima clasificado como cálido seco extremoso, con inviernos moderados y veranos muy calurosos y secos, elevando la temperatura ambiente hasta $48^{\circ} \mathrm{C}$ en verano. Para los cálculos de $\mathrm{R}$, se utilizó la temperatura sol aire, que representa la temperatura de la superficie exterior del muro, expuesta a la radiación solar y aire, para la ciudad de Hermosillo, Sonora, en la latitud geográfica $29.04^{\circ}$ y un meridiano local de $110.58^{\circ}$.

En la figura 1 se muestran los resultados del análisis teórico, donde aparecen los valores de la resistencia térmica y flujos de calor por $\mathrm{m}^{2}$ para cada hora de un día típico de verano, observándose variaciones de $\mathrm{R}$ a lo largo del día de 0.15 a $0.19{ }^{\circ} \mathrm{Cm}^{2} / \mathrm{W}$ que representa una variación del $22 \%$, debida a los fenómenos convectivo y radiativo en la cavidad (Borbón et al., 2009).

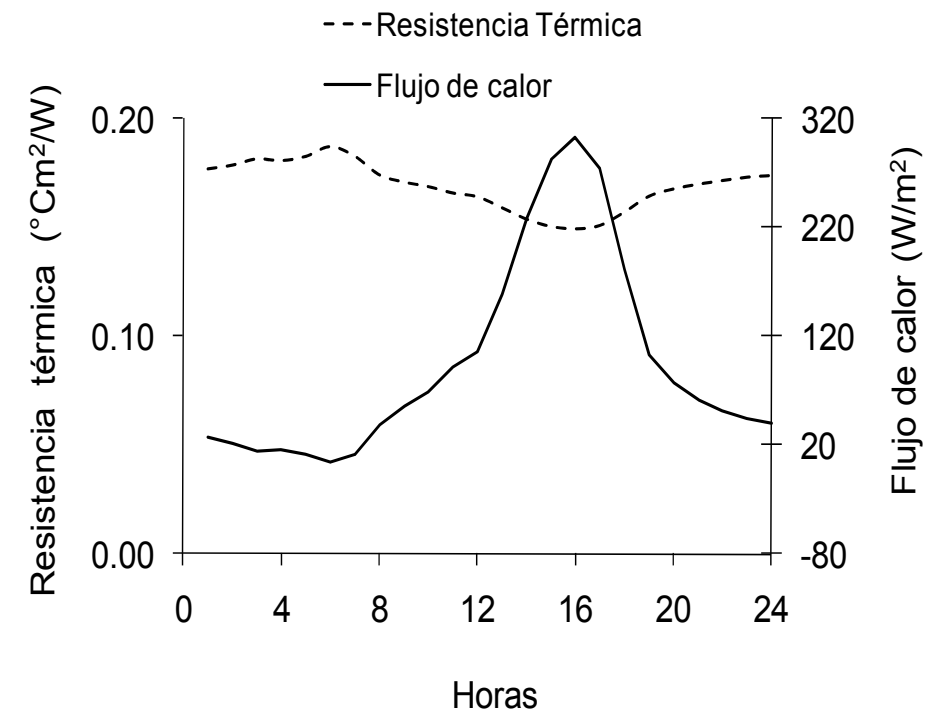

Fig. 1: Resistencia térmica y flujos de calor

A partir de este planteamiento, se diseña y construye un muro de pruebas, para medir las variables que permitan calcular $\mathrm{R}$ de manera experimental. Se consideran para el diseño, las mismas características físicas y dimensionales del bloque, así como condiciones de operación similares a las del modelo. Se utilizan equipos especializados de medición y control de temperaturas, así como sensores de precisión y un detallado sistema constructivo e instalaciones, llevado a cabo bajo supervisión, en condiciones de laboratorio.

\section{DISPOSITIVO EXPERIMENTAL}

Se presenta el diseño de un dispositivo de pruebas para obtener la resistencia térmica en un muro de bloques de concreto, basado en el funcionamiento de placa caliente ASTM 177.Se establece que las pruebas serán bajo estado estacionario, en una pared vertical plana, con un flujo de calor controlado, arreglado de tal forma que suministre un flujo unidireccional y uniforme, creando un diferencial de temperaturas en la pared para diferentes temperaturas de operación y condiciones de frontera adecuadas.

\section{Descripción del dispositivo de pruebas}

En la figura 2 se observa el esquema del dispositivo experimental, que consiste en un gabinete de pruebas constituido por un muro, compuesto de bloques de concreto hueco, y dos paneles de plástico que actúan como intercambiadores de calor, formando una unidad de calentamiento y otra 
de enfriamiento a los lados del muro. La temperatura del agua que recircula por estos paneles, se controla mediante 2 equipos automáticos, uno de calentamiento (calefactor) y otro de enfriamiento (chiller). Se instalan medidores de flujo de agua para cada uno de los intercambiadores, así como sensores de temperatura en las superficies del muro y en las entradas y salidas del fluido (termopozos), conectados a un adquisidor de datos. El sistema está interconectado por medio de tuberías y válvulas de control. El muro y las tuberías se cubren con material aislante, para evitar pérdidas de calor al medio circundante y alcanzar el estado permanente. La figura 3 muestra una vista en planta del sistema.

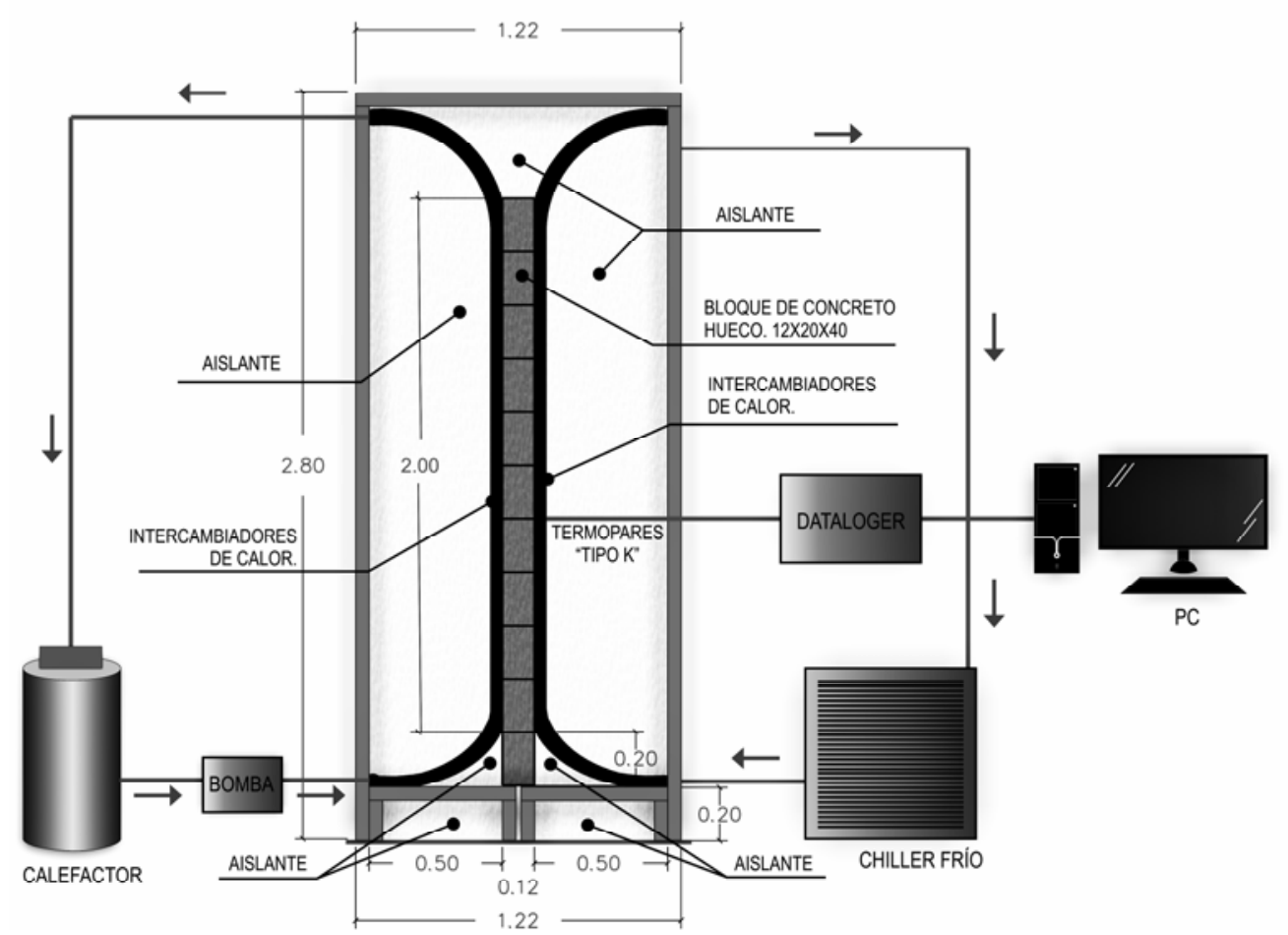

Fig. 2: Esquema del muro de pruebas.

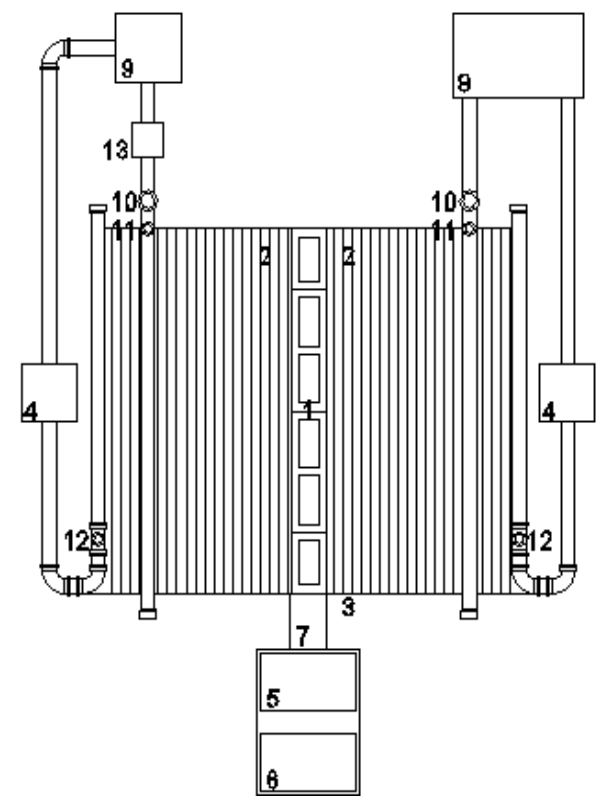

1.- Muro de bloques

2.- Colectores de plástico (panel)

3 - Base de madera

4.- Medidores de flujo

5.- Adquisidor de datos

6.- Equipo de cómputo

7.- Termopares

8.- Equipo de enfriamiento

9.- Equipo de calentamiento

10.- Rotámetros

11.- Termopozos entradas

12.- Termopozos salidas

Fig. 3: Vista en planta del sistema 


\section{Instalaciones}

El área de trabajo donde se construyó el muro es de $40 \mathrm{~m}^{2}$ de superficie, con las instalaciones hidráulicas y eléctricas necesarias para los requerimientos de la prueba.

La temperatura del laboratorio donde se encuentra el muro se mantiene entre 22 a $25^{\circ} \mathrm{C}$ y entre 30 y $35 \%$ de humedad relativa, durante los períodos de prueba.

\section{Variables de medición}

La resistencia térmica del muro se determina en base a las mediciones de temperatura de superficies exteriores del muro y de las temperaturas de entrada y salida del agua de los colectores, en ${ }^{\circ} \mathrm{C}$, así como de los flujos másicos en $\mathrm{l} / \mathrm{min}$, como se describe a continuación.

Temperaturas de superficie del muro exterior lado derecho (ED).

Temperaturas de superficie del muro exterior lado izquierdo (EI).

Temperatura de entrada agua fría.

Temperatura de salida agua fría.

Temperatura de entrada agua caliente.

Temperatura de salida agua caliente.

Flujo agua fría.

Flujo agua caliente.

\section{Clasificación y ubicación de bloques y sensores}

Los bloques y los puntos de medición, fueron identificados y etiquetados. En la figura 4 se muestran los puntos de ubicación de los sensores que se colocaron centrados con respecto a las dimensiones del bloque.

La misma configuración se utiliza en un arreglo simétrico para el lado exterior izquierdo (EI) que representa la parte opuesta del muro.

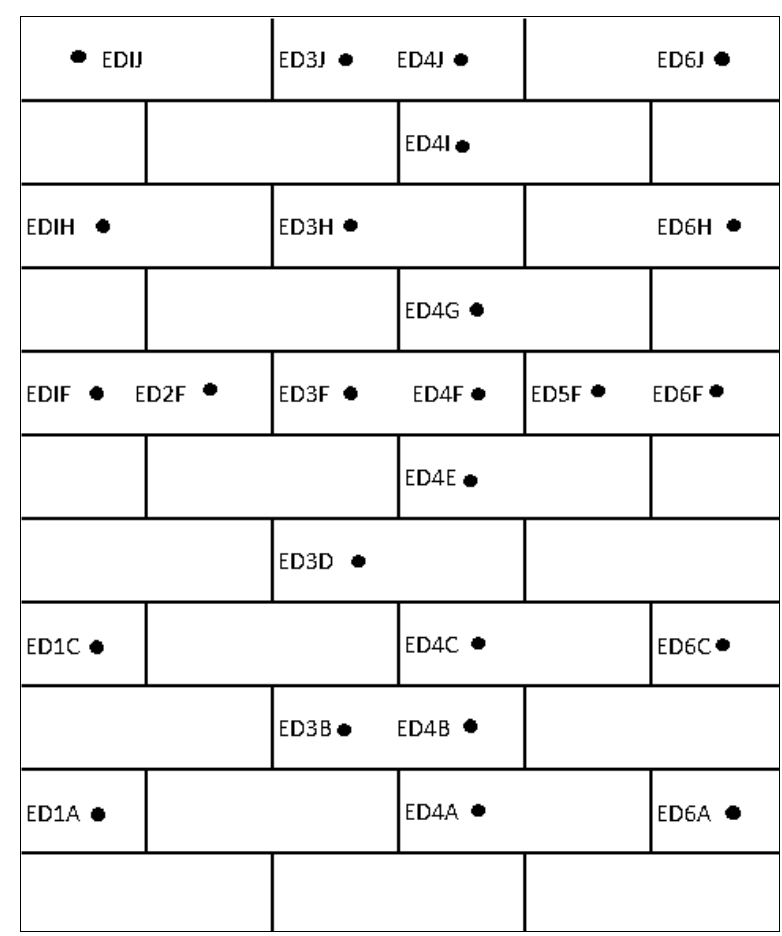

Fig. 4: Ubicación de termopares lado derecho 


\section{Gabinete de pruebas}

La estructura donde se asienta el muro, es una base de madera con elementos verticales y horizontales de sujeción. El muro está elaborado con 25 bloques completos de medida nominal $0.12 \times 0.20 \times 0.40 \mathrm{~m}$ con dos cavidades por bloque de $0.07 \times 0.164 \times 0.20 \mathrm{~m}$, como el que se muestra en la figura 5 , y de 10 medios bloques de medida nominal $0.12 \times 0.20 \times 0.20 \mathrm{~m}$. La altura del muro es de $2.0 \mathrm{~m} \times 1.20 \mathrm{~m}$ de ancho y $0.12 \mathrm{~cm}$ de espesor, basado en la altura de una vivienda tipo. Se forman cavidades alargadas con una relación de aspecto de 28. Los bloques están asentados con un pegamento epóxico, es decir, no se utiliza mortero, por lo tanto no se considera su efecto en la resistencia térmica.

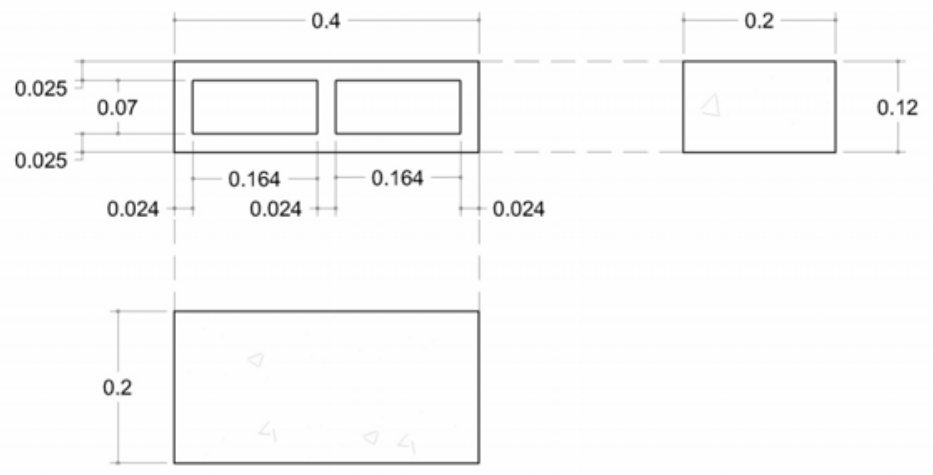

Fig. 5: Dimensiones del bloque

El bloque a utilizar para la construcción del muro, proviene de un mismo banco, que cumple con la norma NMX-C-038 y NMX-C-036, referente a dimensiones y resistencia a la compresión. Estos deberán presentar superficies lisas y homogéneas para asegurar el máximo contacto térmico entre el bloque y las placas, y sin contenido de humedad, para lo cual fueron secados en horno durante 24 horas a $100^{\circ} \mathrm{C}$, eliminando parte del efecto que pudiera causar en la conductividad térmica del material.

El sistema se aísla de la temperatura del medio circundante para alcanzar el estado permanente así como un flujo unidireccional, se utiliza material aislante en las tuberías. En el muro los espesores de material aislante no son menores de $0.25 \mathrm{~m}$.

\section{Instrumentos y equipos}

Intercambiadores de calor.- Se utilizan dos colectores solares de plástico flexible, que son absorbedores de polipropileno con protección UV de 1.30 × 3.05 m PHC-40 EXUN (Línea plástico), que actúa como un intercambiador de calor con el muro, colocado de tal manera que queden adheridos al muro en toda la superficie en sus dos caras, separados en la parte inferior y superior, para evitar el intercambio de calor entre placas.

La selección de los equipos de control de temperatura se hizo de acuerdo a los resultados teóricos, donde se calculó la cantidad de calor que se requiere retirar por $\mathrm{m}^{2}$ de muro, (por lo menos 780 Wats a $0^{\circ} \mathrm{C}$ ) cubriendo los requerimientos para las condiciones de operación a las que será sometido de manera experimental.

Equipo enfriador.- Se utiliza un equipo enfriador (Chiller) para la parte fría del muro, con rango de operación entre -10 y $40^{\circ} \mathrm{C}$, con un porcentaje de incertidumbre de $\pm 0.1^{\circ} \mathrm{C}$.

Equipo calefactor.- Para la zona de calentamiento, se utilizó un equipo recirculador de agua con resistencia, y capacidad de calentar hasta $80^{\circ} \mathrm{C}$, con una incertidumbre de $\pm 0.5^{\circ} \mathrm{C}$. Se utilizó una bomba de $0.5 \mathrm{HP}$ para la recirculación de agua, que trabaja acoplada con este equipo de control de temperatura. 
Medidor de flujo.- Los equipos medidores de flujo son para gastos no mayores de $18 \mathrm{l} / \mathrm{min}$ y presiones bajas. Se utiliza un flujómetro con incertidumbre de $\pm 1 \%$.

Rotámetro de área variable.- Este instrumento de medición, de operación manual, se coloca para tener un control en la estabilidad de los flujos de agua y una referencia en la medición.

Para la instrumentación de los bloques, se utilizan termopares tipo k calibre 36 con una incertidumbre de $0.75 \%$, estos de diferentes longitudes, de acuerdo al diseño, a cada lado del muro, adheridos con un pegamento de baja resistencia térmica.

Adquisidor de datos.- Se utiliza un sistema de adquisición de datos con 4 tarjetas CR-723T Analog Input Card con RTD de catorce canales cada una. Se utiliza el software Logger Net 3.2, que permite monitorear el sistema en tiempo real.

\section{Procedimiento constructivo}

Se construye una base de madera para desplantar el muro con los bloques instrumentados con termopares. Se colocan los colectores y tuberías en los equipos recirculadores de agua y de control de la temperatura del fluido, así como los instrumentos de medición de flujos, todos conectados al adquiridor de datos. Finalmente el sistema es cubierto con material aislante, fabricando volúmenes de siluetas para placas aislantes de poliestireno de densidad igual a $16 \mathrm{~kg} / \mathrm{m}^{3}$. El sistema cuenta con válvulas de desagüe para el llenado, vaciado y cebado del sistema antes de iniciar cada prueba. La figura 6 muestra el dispositivo de medición.

\section{MONITOREO}

Las temperaturas de operación del sistema se establecen considerando variaciones en los lados del muro, para crear diferenciales de temperatura que sirvan para analizar el comportamiento de $\mathrm{R}$ y sus variaciones.

Se colocan 25 sensores de temperatura de cada lado del muro, haciendo un total de 50 termopares, mas 2 de entrada y salida de agua fría, así como 2 adicionales de entrada y salida de agua caliente. Se suman también dos sensores que corresponden a los flujos de agua caliente y fría, haciendo un total de 56, conectados al sistema de adquisición de datos CR7, bajo una programación específica, con el software Loggernet 3.0.

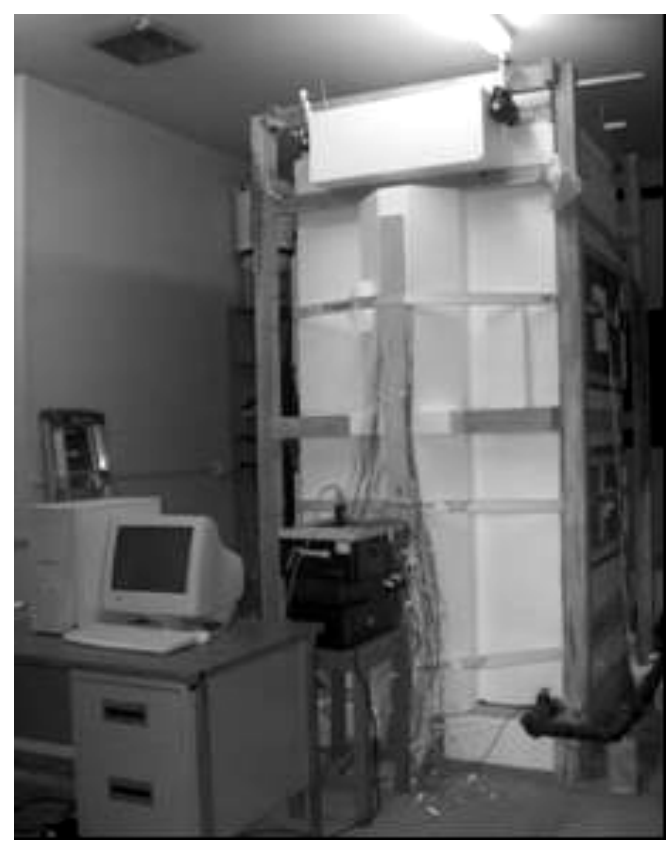

Fig. 6: Gabinete de pruebas 
El monitoreo se inicia con el sistema a temperatura ambiente, variando las temperaturas de operación de los equipos, como se muestra en la tabla 1, para obtener lecturas para cada $\Delta \mathrm{T}$.

Tabla 1: Temperaturas de operación de los equipos.

\begin{tabular}{|c|c|c|c|}
\hline Lecturas & Muro frio & Muro cal & $\Delta \operatorname{Top}\left({ }^{\circ} \mathrm{C}\right)$ \\
\hline 1 & 25 & 45 & 20 \\
\hline 2 & 20 & 45 & 25 \\
\hline 3 & 25 & 55 & 30 \\
\hline 4 & 10 & 45 & 35 \\
\hline 5 & 25 & 65 & 40 \\
\hline 6 & 25 & 70 & 45 \\
\hline 7 & 10 & 70 & 60 \\
\hline
\end{tabular}

\section{Mediciones en estado permanente}

Las temperaturas del agua y de las superficies del muro, para la parte fría y caliente, tienden a la estabilización, alcanzando esta en aproximadamente 6 horas en cada cambio de $\Delta T_{\text {op }}$.

En cada cambio de temperatura en los equipos de control, se espera a que estas se estabilicen en la respuesta del muro, para grabar los datos que se utilizan para los cálculos de q y $R$. Los tiempos de la estabilización del sistema pueden variar dependiendo de las temperaturas iniciales en el muro y las que tienen que alcanzar, y se establecen cuando la diferencia máxima en las lecturas es de $\pm 0.1^{\circ} \mathrm{C}$.

La figura 7 muestra una de las corridas de medición, donde se observa la tendencia de las líneas de temperatura de la superficie de los muros a la estabilización. Las líneas superiores, cercanas los $45^{\circ} \mathrm{C}$ representan la temperatura de entrada y salida del agua caliente, y las cercanas a los $40^{\circ} \mathrm{C}$ representan las temperaturas del muro del lado caliente. Las líneas ubicadas cerca de los $25^{\circ} \mathrm{C}$ representan las temperaturas de entrada y salida del agua, controlada por el enfriador y las cercanas a los $30^{\circ} \mathrm{C}$ las temperaturas del muro frío.

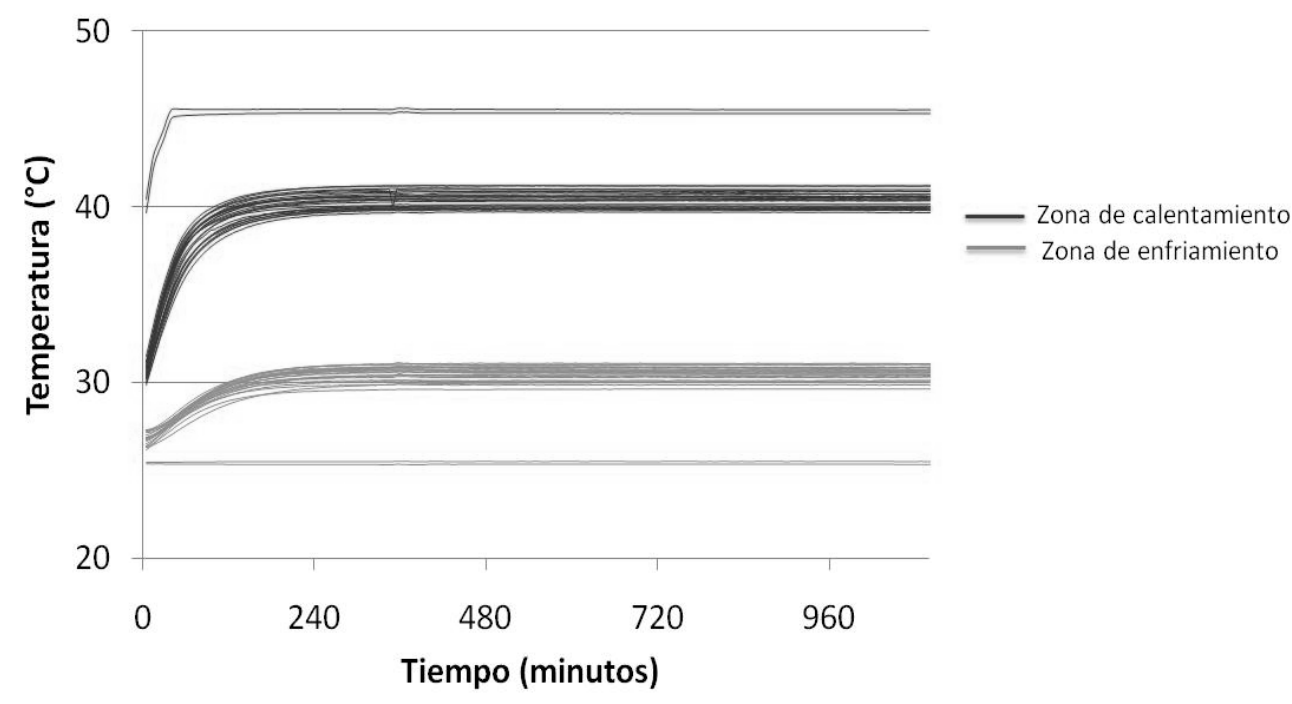

Fig. 7: Estabilización de temperaturas en el flujo y el muro.

Se observa que la distribución de las temperaturas en el muro no son uniformes, teniendo una variación aproximada de $2^{\circ} \mathrm{C}$; esta desviación se debe a que el sistema de placas caliente y fría no tiene un contacto total con la superficie del muro, ni la superficie del muro es lo suficientemente homogénea para obtener una distribución de temperaturas más uniforme. 


\section{RESULTADOS Y DISCUSIÓN}

Para el cálculo de q y $\mathrm{R}$, se consideran los promedios de las temperaturas superficiales a cada lado del muro, así como las temperaturas y los gastos de los flujos.

La tabla 2 muestra los datos de las corridas correspondientes a cada $\Delta T_{\text {op. }} T_{E D}$ describe el valor promedio de la temperatura en el lado derecho del muro, que corresponde a la zona de enfriamiento; $\mathrm{T}_{\mathrm{El}}$ corresponde a la temperatura promedio del muro del lado exterior izquierdo de la zona de calentamiento. $T_{E F} Y T_{S F}$ corresponden a la temperatura de entrada y salida de agua fría. $T_{E C} Y T_{S C}$ corresponden a las temperaturas de entrada y salida del agua caliente y $F_{1}$ y $F_{2}$ a los flujos de agua en $\mathrm{l} / \mathrm{m}$.

Tabla 2.- Resumen de datos resultado de las corridas experimentales.

\begin{tabular}{|c|c|c|c|c|c|c|c|c|c|}
\hline Lect & $\Delta \mathrm{T}_{\mathrm{OP}}$ & $\mathrm{T}_{\mathrm{ED}}$ & $\mathrm{T}_{\mathrm{El}}$ & $\mathrm{T}_{\mathrm{EF}}$ & $\mathrm{T}_{\mathrm{SF}}$ & $\mathrm{T}_{\mathrm{EC}}$ & $\mathrm{T}_{\mathrm{SC}}$ & $\mathrm{F}_{1}$ & $\mathrm{~F}_{2}$ \\
\hline 1 & 20 & 28,76 & 42.34 & 25.37 & 25.56 & 45.55 & 45.35 & 14.17 & 14.78 \\
\hline 2 & 25 & 24.72 & 41.60 & 20.36 & 20.59 & 45.58 & 45.31 & 14.03 & 14.83 \\
\hline 3 & 30 & 30.57 & 50.82 & 25.39 & 25.66 & 55.56 & 55.21 & 14.09 & 15.25 \\
\hline 4 & 35 & 16.76 & 40.07 & 10.32 & 10.66 & 45.51 & 45.16 & 13.75 & 15.05 \\
\hline 5 & 40 & 32.41 & 59.34 & 25.35 & 25.75 & 65.41 & 64.92 & 14.07 & 14.10 \\
\hline 6 & 45 & 33.30 & 63.46 & 25.34 & 25.76 & 70.20 & 69.62 & 14.07 & 14.11 \\
\hline 7 & 60 & 21.73 & 61.56 & 10.33 & 10.93 & 70.30 & 69.63 & 13.69 & 15.52 \\
\hline
\end{tabular}

Con la información de la tabla 2, y las expresiones (1) y (2) se realizan los cálculos para obtener los valores de resistencia térmica y flujos de calor.

$q=G \rho C p \Delta T$ flujo

$R=\frac{\Delta \text { Tmuro }}{q A}$

Donde $\mathrm{G}$ es el caudal en $(\mathrm{l} / \mathrm{m})$, $\rho$ es la densidad del agua de $998\left(\mathrm{~kg} / \mathrm{m}^{3}\right)$ y Cp el calor específico del agua de $4182\left(\mathrm{j} / \mathrm{kg}{ }^{\circ} \mathrm{C}\right)$. Se considera $\Delta \mathrm{T}_{\text {flujo }}$ como la diferencia de temperaturas del flujo de entrada y salida del agua de los intercambiadores de calor.

Para la expresión (2) se considera $\Delta \mathrm{T}_{\text {muro }}$ como la diferencia de temperaturas entre las superficies del muro en estado estable y un área de $2.4 \mathrm{~m}^{2}$ de superficie, siendo $\mathrm{T}_{\text {prom, }}$ el promedio de la temperatura de la parte caliente y fría del muro en estado estable.

La tabla 3 muestra los valores calculados para cada lectura, correspondientes al flujo de calor q, los diferenciales de temperatura del muro en estado estacionario, la temperatura promedio del lado frio y caliente, así como los valores de la resistencia térmica.

Tabla 3: Flujos de calor y resistencias térmicas del muro experimental.

\begin{tabular}{|c|c|c|c|c|}
\hline Corrida & $\mathrm{q}(\mathrm{W})$ & $\Delta \mathrm{T}_{\text {muro }}\left({ }^{\circ} \mathrm{C}\right)$ & $\mathrm{T}_{\text {prom }}\left({ }^{\circ} \mathrm{C}\right)$ & $R\left({ }^{\circ} \mathrm{Cm}^{2} / \mathrm{W}\right)$ \\
\hline 1 & 187.28 & 13.58 & 35.55 & 0.174 \\
\hline 2 & 224.47 & 16.88 & 33.16 & 0.180 \\
\hline 3 & 269.95 & 20.05 & 30.71 & 0.178 \\
\hline 4 & 325.20 & 23.31 & 28.42 & 0.172 \\
\hline 5 & 391.49 & 26.93 & 45.88 & 0.165 \\
\hline 6 & 411.06 & 30.16 & 48.38 & 0.176 \\
\hline 7 & 571.37 & 39.83 & 41.65 & 0.167 \\
\hline
\end{tabular}


En los resultados se muestran variaciones, que pueden obedecer a varios factores, sin embargo eliminando las que se deben a la humedad del material y a la ausencia de mortero, queda por considerar que los coeficientes de radiación de los huecos de los bloques que dependen del acabado superficial, pueden variar, modificando las propiedades aislantes del material.

\section{Cálculo de incertidumbre}

Se calcula la incertidumbre experimental para los datos medidos, utilizando una expresión diferencial que relaciona los resultados de las mediciones, declarando el grado de exactitud de los instrumentos de acuerdo a las especificaciones del fabricante. El resultado $\mathrm{R}$ es una función dada de las variables independientes $x_{1}, x_{2}, x_{3}, \ldots, x_{n}$, por lo tanto, $R=R\left(x_{1}, x_{2}, x_{3}, \ldots, x_{n}\right)$ (Holman, 1987).

Sea $W_{R}$ la incertidumbre en el resultado y $w_{1}, w_{2}, \ldots w_{n}$ las incertidumbres en las variables independientes. Si las incertidumbres en las variables independientes tienen las mismas probabilidades entonces las incertidumbres en el resultado según estas probabilidades está dada por:

$w_{R}=\left[\left(\frac{\partial R}{\partial x_{1}} w_{1}\right)^{2}+\left(\frac{\partial R}{\partial x_{2}} w_{2}\right)^{2}+\ldots+\left(\frac{\partial R}{\partial x_{n}} w_{n}\right)^{2}\right]^{1 / 2}$

\section{Cálculo de la incertidumbre para q}

La ecuación (3) se deriva parcialmente con respecto al caudal y a la temperatura de entrada y salida del agua fría. Se calculan los valores para las derivadas con los datos de la tabla 4.

Tabla 4.- Valores para el cálculo de de la incertidumbre experimental

\begin{tabular}{|c|c|c|}
\hline Variable & Valores & Unidades \\
\hline Caudal (G) & 14.17 & $\mathrm{I} / \mathrm{min}$ \\
\hline Densidad del agua $(\rho)$ & 0.998 & $\mathrm{~kg} / \mathrm{l}$ \\
\hline Temperatura de entrada del agua fría (Tef) & 25.56 & ${ }^{\circ} \mathrm{C}$ \\
\hline Temperatura de salida del agua fría (Tsf) & 25.37 & ${ }^{\circ} \mathrm{C}$ \\
\hline$\Delta T$ del agua fría (Tsf -Tef) & 0.19 & ${ }^{\circ} \mathrm{C}$ \\
\hline Calor específico del agua (Cp ) & 4182 & joul \\
\hline
\end{tabular}

De acuerdo a la ecuación 3 , se calculan las incertidumbres de las variables independientes $W_{G}, W_{t c} y$ $\mathrm{W}_{\mathrm{tf}}$, correspondientes a la incertidumbre del flujómetro y de los sensores de temperatura de entrada y salida del agua (zona de enfriamiento) respectivamente, y lo establecido en los manuales de operación de $0.75 \%$ de error para los termopares y de $1 \%$ para el medidor de flujo, dando como resultado un error de $\pm 3.75 \%$.

\section{Comparación de resultados teóricos y experimentales}

La figura 8 muestra los valores de $\mathrm{R}$, tanto experimentales como numéricos para las mismas condiciones de $\Delta \mathrm{T}$, observando que ambos resultados están dentro del rango de precisión experimental calculado, lo que valida el modelo teórico propuesto. Sin embargo aparece un punto donde esta concordancia no se presenta por lo que se requería mayor profundidad para explicar este caso. Ambos muestran una tendencia a la disminución de $\mathrm{R}$ con respecto al aumento de $\Delta \mathrm{T}$, sin embargo, esta variación parece estar condicionada a otro parámetro, ya que los valores de $\Delta T$ más grandes no muestran la disminución del valor de $\mathrm{R}$ que se podría esperar. 


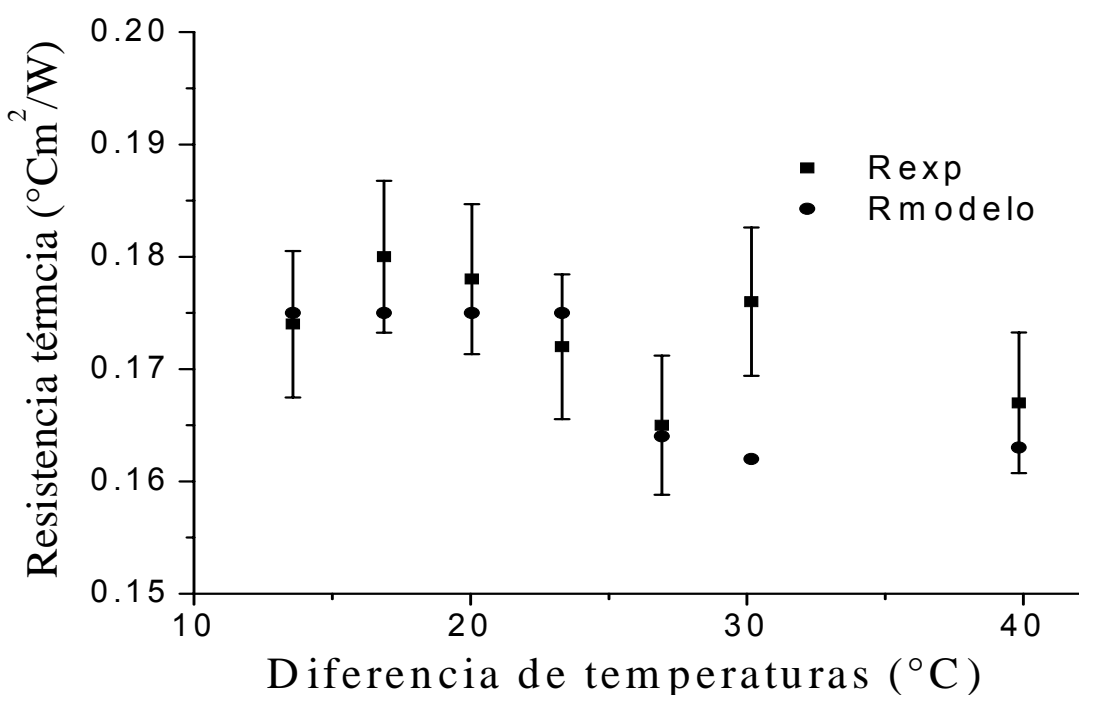

Fig. 8: Resultados experimentales y validación del modelo teórico.

\section{Ecuación de ajuste para la resistencia térmica}

Dada la concordancia de los resultados teóricos y experimentales, se considera el modelo teórico como útil y confiable para llevar a cabo un estudio paramétrico y proponer una expresión empírica que permita calcular el valor de $\mathrm{R}$ para diferentes condiciones de operación, en un muro de bloques de concreto hueco, de medidas nominales $0.12 \times 0.20 \times 0.40 \mathrm{~m}$, con dos cavidades de $0.07 \times 0.164 \times$ $0.20 \mathrm{~m}$, para lo cual se realiza una correlación de datos de los valores obtenidos de la corrida del modelo teórico para condiciones de invierno (24 horas) y verano (24 horas) de los días seleccionados para el análisis, además de algunos datos adicionales que no corresponden a estas condiciones, tomados como posibles puntos intermedios en el rango de operación de las temperaturas, haciendo un total de 67 datos. Además de la variable $X=\Delta T$, se toma en cuenta la temperatura promedio de las superficies del muro $Y=T_{\text {prom }}$ y $Z=R$, considerando esta última como la variable dependiente.

Se considera un rango de $\Delta \mathrm{T}$ de $0.86^{\circ} \mathrm{C}$ a $45.81^{\circ} \mathrm{C}$ y un rango de $\mathrm{T}_{\text {prom }}$ de $12.75^{\circ} \mathrm{C}$ a $47.60^{\circ} \mathrm{C}$ para la correlación, que cubre las condiciones de operación de invierno y verano en clima cálido seco extremoso.

Realizando un ajuste de los resultados experimentales y teóricos encontrados, se encuentra la expresión empírica que se describe a continuación.

$$
R=\frac{1}{4.78+0.142 \sqrt{|\Delta T|}+0.021 \overline{T_{\text {prom }}}}
$$

$$
\text { Para } \quad 0.86 \leq|\Delta T| \leq 45.81 \quad \text { Y } \quad 12.75 \leq T_{p} \leq 47.60
$$

Donde $\mathrm{R}=$ Resistencia térmica

$T_{\text {prom }}=\frac{T_{c}+T_{f}}{2} \quad \mathrm{~T}_{\mathrm{c}}=$ Temperatura caliente en el muro y $\mathrm{T}_{\mathrm{f}}=$ Temperatura fría en el muro

$|\Delta T|=$ Valor absoluto de los diferenciales de temperatura entre la superficie caliente y fría del muro. 
La expresión (4) presenta un ajuste a los datos con un error máximo porcentual de $0.67 \%$ y un factor de correlación de $r^{2}=0.992$. Lo que nos sugiere que las variables independientes para el valor de $R$ son tanto la diferencia de temperatura como la temperatura promedio.

La figura 9 muestra la superficie de respuesta de las variables correspondientes a $\Delta T, T_{\text {prom }}$ y resistencia térmica, donde se observan los puntos correspondientes a las condiciones de invierno y verano, analizadas, así como puntos intermedios de estos valores.

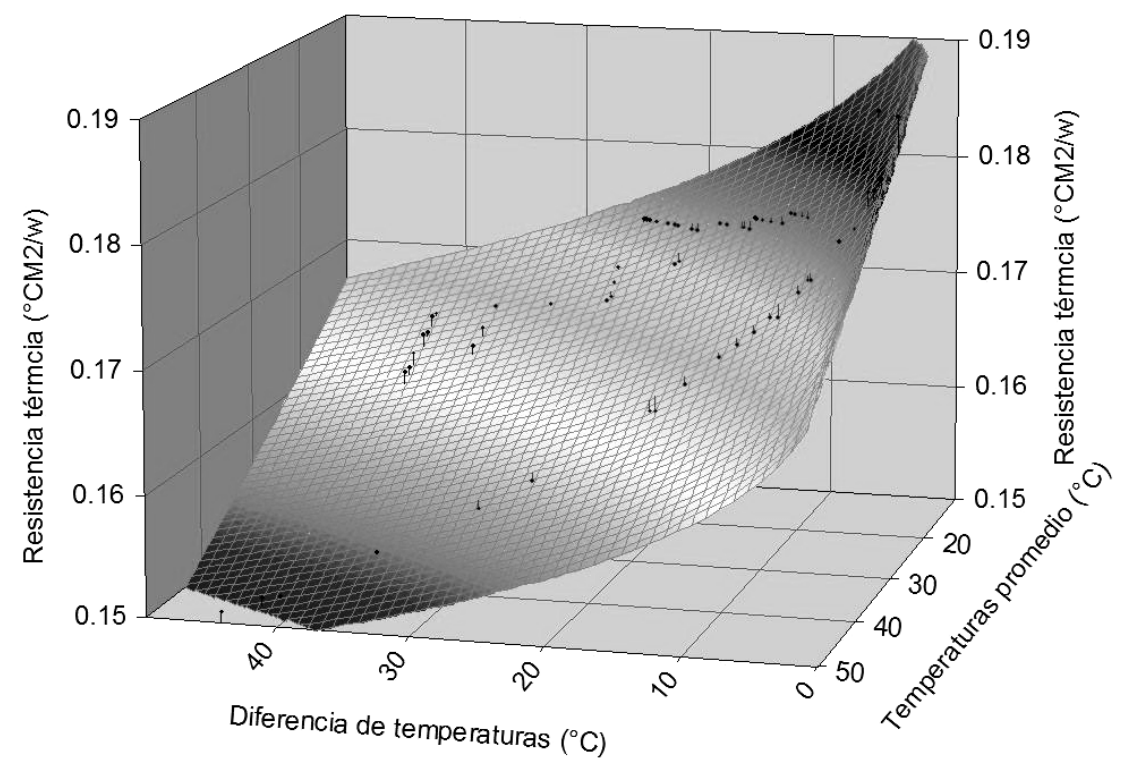

Fig. 9: Superficie de respuesta de la ecuación de ajuste para R.

La correlación empírica encontrada muestra que tanto la diferencia de temperatura como la temperatura promedio están relacionadas con el valor de la R. Esto se explica porque la diferencia de temperatura aumenta la facilidad de transferir calor dentro de la cavidad motivado principalmente por la convección y la radiación, mecanismos que están acoplados, uno en forma lineal (convección) y otro con términos a la cuarta (radiación), pero a su vez la temperatura promedio del fluido, en este caso el aire, afecta las propiedades termodinámicas del mismo lo que a su vez influye en el transporte convectivo.

\section{CONCLUSIONES}

Los resultados experimentales muestran concordancia con los obtenidos teóricamente, lo que sugiere que las suposiciones hechas son suficientemente correctas para ser usadas en estudios posteriores. Dada la similitud entre los resultados teóricos y experimentales para el valor de $R$, se concluye que el modelo matemático es útil para predecir el comportamiento de la transferencia de calor en el bloque hueco, considerando radiación, convección y conducción de manera acoplada. Se corrobora también mediante los dos modelos la variabilidad de $\mathrm{R}$ con respecto a las variables asociados a los cambios en la temperatura, tomando en cuenta los $\Delta T$ y temperaturas promedio en el muro, expresadas en la construcción de una superficie de respuesta que se ajusta a una sencilla expresión empírica, tomando como variables independientes la diferencia de temperatura y la temperatura promedio del muro, con un alto factor de correlación.

El que se haya podido lograr el ajuste de los datos de $\mathrm{R}$, sugiere que las variables independientes seleccionadas son correctas y se explica porqué la diferencia de temperatura afecta a ambos mecanismos (radiación-convección), pero además que la temperatura promedio influye en las propiedades termodinámicas del aire confinado en la cavidad del bloque.

Con la obtención de la expresión de la resistencia térmica en función de la diferencia de temperatura y la temperatura promedio, se puede estimar con buena concordancia los valores de la resistencia 
térmica del muro de bloque, lo cual simplifica enormemente la obtención de este valor y facilita su integración a paquetes de simulación de balances energéticos en edificaciones.

\section{NOMENCLATURA}

$\sigma \quad$ Densidad del agua $\mathrm{kg} / \mathrm{m}^{3}$.

$\mathrm{Cp} \quad$ Calor específico del agua $\left(\mathrm{j} / \mathrm{kg}{ }^{\circ} \mathrm{C}\right)$

$\Delta \mathrm{T} \quad$ Diferencia de temperatura ${ }^{\circ} \mathrm{C}$.

G Caudal en $1 / \mathrm{min}$.

$\Delta T_{\text {flujo }} \quad$ Diferencia de temperatura del fluido ${ }^{\circ} \mathrm{C}$.

A Aérea del muro $\mathrm{m}^{2}$.

$W_{R} \quad$ Incertidumbre en la medición del flujo.

$W_{G} \quad$ Incertidumbre del flujómetro.

$\Delta \mathrm{T}_{\text {op }} \quad$ Diferencia de temperaturas de operación de los equipos.

q Flujo de calor $\mathrm{w} / \mathrm{m}^{2}$.

$\mathrm{F}_{1} \quad$ Flujo de agua fría $\mathrm{l} / \mathrm{min}$.

$\mathrm{F}_{2} \quad$ Flujo de agua caliente I /min.

$\mathrm{R} \quad$ Resistencia térmica ${ }^{\circ} \mathrm{Cm}^{2} / \mathrm{w}$.

$\mathrm{T}_{\mathrm{EC}} \quad$ Temperatura entrada de agua caliente ${ }^{\circ} \mathrm{C}$.

$\mathrm{T}_{\mathrm{SC}} \quad$ Temperatura salida de agua caliente ${ }^{\circ} \mathrm{C}$.

$\mathrm{T}_{\mathrm{SF}} \quad$ Temperatura salida de agua fría ${ }^{\circ} \mathrm{C}$.

$\mathrm{T}_{\mathrm{EF}} \quad$ Temperatura entrada de agua fría ${ }^{\circ} \mathrm{C}$.

$\mathrm{T}_{\mathrm{El}} \quad$ Temperaturas de superficie del muro exterior lado izquierdo en estado estable ${ }^{\circ} \mathrm{C}$.

$\mathrm{T}_{\mathrm{ED}} \quad$ Temperaturas de superficie del muro interior lado derecho en estado estable ${ }^{\circ} \mathrm{C}$.

$\mathrm{T}_{\text {prom }} \quad$ Temperatura promedio en el muro $\left({ }^{\circ} \mathrm{C}\right)$.

$\Delta \mathrm{T}_{\text {muro }}$ Diferencia de temperaturas en el muro en estado estable ${ }^{\circ} \mathrm{C}$.

\section{AGRADECIMIENTOS}

A la División de Ingeniería de la Universidad de Sonora, México, por los apoyos brindados para la publicación de este artículo y a la Universidad Autónoma de Baja California, México.

\section{REFERENCIAS}

Al-Jabri K.S., Hago A.W., Al-Nuaimi A.S., Al-Saidy A.H., Concrete blocks for thermal insulation in hot climate, Cement and Concrete Research: 35, 1472-1479 (2005).

Anderson, B. R., On the Calculation of the U-value of Walls Containing Slotted Bricks or Blocks, Building and Environment: 16 (1), 41- 50 (1981).

Aviram, D.P., A.N. Fried, y J.J. Roberts, Thermal Properties of a Variable Cavity Wall, Building and Environment: 36, $1057-1072$ (2001).

Borbón, A.C., Cabanillas, E., Pérez, J., Modelación y Simulación de la Transferencia de Calor en Muros de Bloque de Concreto Hueco, Información Tecnológica: ISSN: 0718-0764 (En línea), 21(3), www.citchile.cl (2010).

Chavez Y., Wilson R., A second degree approximation for the calculation of the transfer function coefficients for heat conduction through walls, Energy and Buildings: 40 (4), 549-555 (2008).

Del Coz J., Garcia, C., Betegón M., Prendes G., Analysis and optimization of the heat-insulating light concrete hollow brick walls design by the finite element method, Applied Thermal Engineering, $27(8,9) 1445-1456$ ( 2007). 
Holman, J., Métodos experimentales para Ingenieros, Cuarta edición. MacGraw Hill, México (1987).

Kossecka, E., Kosny J. Three-dimensional conduction z-transfer function coefficients determined from the response factors, Energy and Buildings, 37( 4), 301-310 (2005).

Lacarriere B., Trombe A., y Monchoux F, Experimental Unsteady Characterization of Heat Transfer in a Multi-layer Wall Including Air layers-Application to Vertically Perforated Bricks, Energy and Buildings: 38, 232-237. (2006).

NMX-C-036-ONNCCE, Norma Mexicana, Industria de la Construcción. Bloques tabiques o ladrillos, tabicones y adoquines, Resistencia a la compresión, Método de prueba, 3-6, México (2004).

NMX-C-038-ONNCCE, Norma Mexicana, Industria de La Construcción - Bloques -Tabiques o Ladrillos y Tabicones para uso estructural, 2-6, México (2004).

Ossama A. Abdou, y Kris S. Murali b., The Efect of Air Cells and Mortar Joints on the Thermal Resistance of Concrete masonry Walls, Energy and Buildings: 21, 111-119 (1994).

Standard Test Method for Steady-State Heat Flux Measurement and Thermal Transmission Properties by Means of the Guarded-Hot Plate Apparatus. Designation: C177-97.

Vasile, C., S. Lorente, y B. Perrin, Study of Convective Phenomena Inside Cavities Coupled with Heat and Mass Transfers Through Porous Media- Application to Vertical Hollow Bricks- A first Approach, Energy and Buildings: 28, 229-235 (1998). 